\title{
Ecology of Italian Protura
}

Loris Galli $^{1 *}$, Matteo Capurro ${ }^{1}$, Tony Molyneux ${ }^{2}$, Carlo Torti ${ }^{1} \&$ Matteo Zinni ${ }^{1}$

${ }^{1}$ Dipartimento di Scienze Della Terra, dell'Ambiente e della Vita, Genoa University, Corso Europa 26, I16132, Italy.

${ }^{2}$ Gungahlin College, Canberra, Australia

* Corresponding author. Permanent address: Dipartimento di Scienze Della Terra, dell'Ambiente e della Vita, Genoa University, Corso Europa 26, I16132, Italy.

E-mail address: loris.galli@dipteris.unige.it

\begin{abstract}
The ecology of Protura in Italy (including Corsica) is tentatively described according to original and bibliographical data. Protura are quite common but very rarely abundant: their mean density in soil samples collected in Liguria (NW Italy) was estimated to be $372 / \mathrm{m}^{2}$ (s.d. 657 - max. $2790 / \mathrm{m}^{2}$ in a Holm oak forest). Information from the analyses of habitat and/or elevation of 3448 specimens from 269 collections and 4071 specimens from 295 collections identified to species and genus level, respectively, enabled us to outline Protura assemblages for eight different macro-habitats. The unbalanced sex ratio in favour of females observed in 12 of the dominant species of Acerentomata suggests that (based also on the sperm types known in Protura) a single male can fertilize several females through spermatophores. For one species (Proturentomon minimum) only females were collected, which may suggest the possibility of parthenogenesis in some Protura. An analysis of the phenology and population dynamics of the five dominant species, showed annual cycles with one (Acerentomon microrhinus) or more (A. gallicum, A. italicum, A. maius and Eosentomon transitorium) peaks of reproductive activity. Other ecological remarks (e.g. about the relationship between the amplitude of latitudinal/altitudinal distribution and the ecological distribution of Protura in Italy) are discussed.
\end{abstract}

\section{Keywords}

Italy, Soil, Protura, Habitat, Ecology, Phenology

\section{Introduction}

Protura are small hexapods belonging to the soil mesofauna. They are widely distributed throughout the world with more than 800 species (748 were listed by Szeptycki, 2006), collected in several 
different habitats at elevations ranging from sea level to about $4000 \mathrm{~m}$ [Eosentomon validum, Bujuku Lake, Rwenzori Mountains, Uganda (Nosek, 1975)]. Their distribution seems to be limited by the availability of sufficient soil moisture for plant growth or by the availability of deposits of decaying organic matter (Nosek, 1975). Their vertical distribution is rather limited with higher densities recorded in the first few centimetres of soil.

Protura are generally poorly known and few studies have specifically focused on their ecology. Most field work has involved studying individual habitats and/or small areas (Raw, 1956; Stumpp, 1990; Christian and Szeptycki, 2004; Mitrovski Bogdanović and Blesic, 2011). Only in a few cases has ecological information about Protura been inferred on a large geographical scale based on statistical analyses of national or regional species distribution (e.g. Imadaté, 1974; Szeptycki et al., 2003). At present 42 species have been collected in Italy (Galli et al. 2011) but only sporadic information on the ecological factors that influence their occurrence in different habitats, and about their phenology is available.

In this paper we have analysed demographic and ecological data important in the distribution and population dynamics of Protura in Italy and Corsica. In particular, we have tried to answer the following questions. (Q1) How abundant are Protura? (Q2) Are Protura randomly distributed or does their distribution reflect some pattern? (Q3) Is the abundance of Protura influenced by the local geological substratum, by the dominant plant species, or by a combination of these factors? (Q4) Is there a relationship between single genera/species or assemblages of Protura and habitat/elevation? (Q5) Is there a relationship between the ecology of habitat and the geographical distribution of Protura in Italy? (Q6) Does sex ratio result balanced or unbalanced amongst populations of Protura? And (Q7) what are the annual cycles of the Proturan species found in the study area?

Part of the results of this study was briefly illustrated in Italian by Galli and Zinni (2017).

\section{Material and methods}

\section{Protura collection and identification}

Specimens in the authors' collections were extracted from soil samples into $70 \%$ ethanol using Berlese-Tullgren funnels with $2.5 \mathrm{~mm}$ mesh size for seven days. Newly extracted Protura and museum specimens preserved in $70 \%$ ethanol (after Berlese-Tullgren extraction) were cleared in lactic acid for 24 hours at $40-50^{\circ}$, then mounted on slides in Marc André II medium. Protura were identified to species and life stage levels (prelarva, larva I, larva II, maturus junior, preimago and imago) using an interference contrast microscope (Leica DM LB2), a Leica DFC 295 camera and Leica Application Suite Vers. 3.8. 


\section{Analysis of density}

To answer our first question (Q1), quantitative data obtained from 405 soil samples $\left(2000 \mathrm{~cm}^{3}\right.$, nearly $200 \mathrm{~cm}^{2}$ of surface, $10 \mathrm{~cm}$ depth) collected between 2003 and 2016 from 32 stations in Liguria (NW-Italy, Table 1) by the Italian authors, were used to extrapolate densities of Protura on a surface area of $1 \mathrm{~m}^{2}$.

\section{Table 1}

Sites in Liguria (NW-Italy) analysed for an estimation of the density of Protura. For each habitat the number of sampling localities and the total of collected samples are shown.

\begin{tabular}{|l|r|r|}
\hline \multicolumn{1}{|c|}{ Habitat } & Nr of localities & Nr of samples \\
\hline Mediterranean maquis & 2 & 22 \\
\hline Cork oak forest & 1 & 12 \\
\hline Holm oak forest & 9 & 72 \\
\hline Mixed forest (locust tree dominant) & 2 & 51 \\
\hline Chestnut forest & 3 & 84 \\
\hline Beech forest & 4 & 71 \\
\hline Submontane prairie - bush & 3 & 29 \\
\hline Rocks vegetation & 2 & 20 \\
\hline Pines forest in city Park & 2 & 7 \\
\hline City Park & 3 & 29 \\
\hline Uncultivated meadow & 1 & 8 \\
\hline
\end{tabular}

On such samples, aggregation level was estimated using $J$ index (Ives, 1991):

$$
J=\left\{\left[\sum n_{i}\left(n_{i}-1\right)\right] 1 / N m\right\}-1
$$

Where $n_{i}$ is the number of individuals in the soil core $i, m$ is the mean of $n_{i}$ and $N$ is the total number of individuals across all samples. $J$ measures the proportional increase of individuals encountered by an individual relative to a random or Poisson distribution. $J<0$ describes a uniform distribution; when $J=0$ individuals are randomly distributed, whereas $J>0$ indicates an aggregated distribution (Q2).

Data from three sampling sites among the localities shown in Table 1 were used to evaluate the influence of plant species and/or the substratum on Proturan abundance (Q3). Different coppices of a similar age in sampling sites close to each other, and with the same exposure and elevation range (Ferretti S. \& Galli L., unpubl.) were specially chosen to override the effect of forest management, location and relative climatic differences on soil arthropods communities. On the northern slopes of each of three mountains in the Genoa province, a chestnut (Castanea sativa) and a beech (Fagus sylvatica) forest between 900-1000 and 1100-1300 m elev., respectively, were selected. In 2008, 
seven samples in each site were simultaneously collected every month (in April, June, September and November in chestnut woods; in May, July and October in beech ones). Elevation, geological origin of the soil, granulometry and $\mathrm{pH}$ for each forest were recorded (Table 2).

\section{Table 2}

Elevation, geological origin of the soil, granulometry $(\mathrm{mm})$ and $\mathrm{pH}$ of chestnut and beech forests in three sites within the Genoa province (Liguria, NW-Italy).

\begin{tabular}{|c|c|c|c|c|c|}
\hline Site & Geology & Habitat & Elevation & pH & Granul. \\
\hline Mt Antola & \multirow{2}{*}{ marly limestones } & chestnut & 900 & 7.3 & 0.01 \\
\hline Mt Antola & & beech & 1280 & 7.6 & 0.01 \\
\hline Mt Penna & \multirow{2}{*}{ flysh } & chestnut & 970 & 6.1 & 0.05 \\
\hline Mt Penna & & beech & 1240 & 6.5 & 0.05 \\
\hline Mt Zatta & \multirow{2}{*}{$\begin{array}{l}\text { siltstones and sandstones intercalated with shales } \\
\text { in arkosic and subarkosic composition }\end{array}$} & chestnut & 940 & 5.9 & 0.05 \\
\hline Mit Zatta & & beech & 1170 & 6 & 0.05 \\
\hline
\end{tabular}

Differences in densities of Protura collected in the beech and in the chestnut forests on the same mountain may be attributed to the plant species effect on soil characteristics; on the other hand, differences among numbers of Protura recorded in the same habitat on the three mountains are likely related to the influence of the geological substratum on the local soil.

\section{Analysis of ecological distribution}

Data of Protura from Italy (including Corsica) were analysed. Information was obtained from the original descriptions of species with type localities in Italy and Corsica and from other literature (Nosek, 1973; Condé, 1980; Galli et al., 2011), as well as from the collections of Natural History Museums of Geneva, Genoa and Verona, and of the authors themselves (Tables 3 and 4). Geographical distribution of the collecting localities was outlined according to the geographical units suggested by Biondi et al. (2013) (Fig. 1). Due to the low number and the heterogeneous distribution of samplings, sampling localities were grouped into four macro-units: North (NorthWestern Alps, Eastern Alps, South-Western Alps and Padanian Province), Center (Northern and Central Apennines), South (Southern Apennines and Apulian Province) and Islands (Corsica, Sardinia, Sicily and Tyrrhenian Islands).

The ecological information (habitat and/or elevation) was used to assess ecological distribution of Protura at genus and species level (Q4). To facilitate comparisons with sufficient replication, habitat categories were grouped, where possible, to into the following eight "macro-habitats": Mediterranean maquis (seven collections), Mediterranean sclerophyllous forest (78), basal plane deciduous forest (64), sub-montane deciduous forest (50), montane deciduous forest (49), montane coniferous forest (seven), olive trees (nine), and vineyards (five). Based on the expert advice from 
Dr. G. Barberis (Professor of Botany at the Genoa University) these groupings reflect key differences in habitat, geographical region and elevation. City Parks were not grouped into a macrohabitat since they were too different from each other in terms of plants (exotic plants, Mediterranean coniferous, bushes and meadows, Holm oak woods) and management.

Overall, the data associated with 3448 specimens from 269 collections identified to species level and 4071 specimens from 295 collections identified to genus, were analysed (Table 3).

\section{Table 3}

Distribution of collections in the geographical units in which Italy was divided (including Corsica) with the corresponding number of Protura identified to genus and species level.

\begin{tabular}{|l|l|c|c|c|c|}
\hline \multirow{3}{*}{$\begin{array}{c}\text { Geographical } \\
\text { macrounit }\end{array}$} & \multirow{2}{*}{ Geographical unit } & \multicolumn{2}{|c|}{ Species level } & \multicolumn{2}{c|}{ Genus level } \\
\cline { 2 - 6 } & Collecting sites & Protura & Collecting sites & Protura \\
\hline \multirow{4}{*}{ North } & NW Alps & 10 & 166 & 13 & 218 \\
\cline { 2 - 6 } & E Alps & 11 & 219 & 11 & 259 \\
\cline { 2 - 6 } & SW Alps & 75 & 1326 & 76 & 1509 \\
\cline { 2 - 6 } & Padanian & 24 & 249 & 27 & 260 \\
\hline \multirow{3}{*}{ Center } & N Apen & 95 & 1047 & 110 & 1287 \\
\cline { 2 - 6 } & C Apen & 11 & 77 & 12 & 88 \\
\hline \multirow{3}{*}{ South } & Apulian & 8 & 26 & 9 & 29 \\
\cline { 2 - 6 } & S Apen & 6 & 51 & 6 & 71 \\
\hline \multirow{4}{*}{ Islands } & Tyrrh Isl & 3 & 69 & 8 & 93 \\
\cline { 2 - 6 } & Corsica & 7 & 47 & 18 & 172 \\
\cline { 2 - 6 } & Sardinia & 17 & 153 & 2 & 20 \\
\cline { 2 - 6 } & Sicily & 2 & 18 & & \\
\hline
\end{tabular}

A second round of analysis on the Table 3 data was performed to evaluate whether there was a relationship between the geographical distribution of Protura (estimated based on the number of geographical units in which they were found) and their ecological adaptability (in terms of number of habitats in which they were present) (Q5).

\section{Sex ratio and phenology}

The sex ratio (number of males/number of females), phenology and seasonality of juvenile instars of the dominant species were analysed to obtain detailed information on their demographic structure and population dynamics (Qs 6 \& 7). The whole dataset of 5910 Protura from Italy (and Corsica) identified to species level from literature as well as from the aforementioned collections was used, including specimens from samples lacking detailed information about the sampling locality. In particular, monthly numbers of larva I and larva II were counted together, and for Acerentomidae, the numbers of maturus junior and pre-imago were also combined (in Eosentomidae pre-imago is absent). Distribution of collections during the year was almost uniform (Fig. 3A) with a value significantly lower than the average only in December $\left(\chi^{2}=34.5,11\right.$ d.f., $\left.p<0.01\right)$. Therefore, apart 
from any minimum peak during that month, the monthly numerical fluctuations of individuals can be considered to correspond to real population dynamics in the wild.

\section{Statistical analysis}

The aggregation level of Protura in the 405 soil samples in 32 stations in Liguria was estimated by calculating $J$ index (see Analysis of density). The statistical significance of the differences between Protura numbers in collected soil samples and expected frequencies based on a Poisson distribution $(\lambda=m)$ was assessed using the Chi-square test.

Differences between the numbers of Protura in soils of chestnut and beech woods studied in the Genoa province (see Table 2) were also evaluated using the Chi-square test; those among the three mountains characterized by a different geological substratum were investigated using the Pairwise Mann-Whitney test; and linear regression analysis was performed to estimate the relationship between the number of Protura and $\mathrm{pH}$ (both variables log transformed).

Differences in assemblages among the eight macro-habitats were investigated by means of one-way ANOSIM. SIMPER analysis allowed the identification of taxa responsible for significant differences. Moreover, paired group-clustering based on the Bray-Curtis similarity index (1000 bootstraps) was used to compare such macro-habitats based on the respective Proturan assemblages. To test whether there was an interrelationship between the geographical distribution of Protura and their ecological adaptability, statistical significance of the differences among geographical macrounits (North, Center, South and Islands) in terms of geographical spread (estimated based on the number of geographical units in which they were found) and number of colonized habitats by Protura spp. was analysed using the Kruskal-Wallis test. The relationship between the extent of geographical distribution and the number of colonized habitats was tested using linear regression analysis.

The statistical significance of the differences from the expected value (1) of the species sex ratio was assessed using the Chi-square test.

PAST Software (Paleontological Statistics version 2.17; Hammer et al. 2001) was used to perform all the statistical analyses mentioned above.

\section{Results}

\section{Analysis of density}

The density of Protura in soil samples collected in Liguria ranged between 0 and 2790 specimens $/ \mathrm{m}^{2}$ with a mean value of 372 (s.d. 657), which constitutes, on average, $1.80 \%$ of the total soil arthropods sampled $\left(23233 \pm 19398\right.$ individuals $\left./ \mathrm{m}^{2}\right)(\mathrm{Q} 1)$. 
Frequencies of Protura in examined soil samples showed a "zero-inflated" distribution, with the resultant $\mathbf{J}$ value of 7.64, suggesting aggregation $(\mathrm{Q} 2)$. This was confirmed after we observed a significant difference $\left(\chi^{2}=2816,10\right.$ d.f., $\left.\mathrm{p}<0.01\right)$ when we compared our data distribution with an expected Poisson distribution with the same mean $m$ (4.64 Protura/sample).

A more detailed analysis on the ecological factors influencing abundance of Protura was performed using data collected from soil samples in chestnut and beech forests soil samples on three mountains in the Genoa province (Tab. 2) (Q3). Monthly numbers of Protura recorded in chestnutforested soils were higher than those recorded in beech-forested soils at a level close to statistical significance $\left(\chi^{2}=5.1698,2\right.$ d.f., $\left.p=0.075\right)$. Moreover, in the slightly alkaline, marly limestone soils of Mt Antola, characterized by $0.01 \mathrm{~mm}$ granulometry, Protura densities $\left(50 / \mathrm{m}^{2}\right.$ under chestnuts $40 / \mathrm{m}^{2}$ under beeches) were 3-10 times lower than those recorded in slightly/moderately acidic flysh and siltstones/sandstones soils with a $0.05 \mathrm{~mm}$ granulometry found under beeches and chestnuts on Mt Penna $\left(480 / \mathrm{m}^{2}\right.$ in chestnuts $-270 / \mathrm{m}^{2}$ in beeches) and on Mt Zatta $\left(530 / \mathrm{m}^{2}\right.$ in chestnuts -120 $/ \mathrm{m}^{2}$ in beeches), respectively. The Pairwise Mann-Whitney test demonstrated a close similarity in abundance of Protura between Mt Zatta and Mt Penna, a significant difference between Mt Zatta and Mt Antola $(\mathrm{p}=0.03)$ and an almost significant difference between Mt Penna and Mt Antola ( $\mathrm{p}$ $=0.08$ ). It was possible to define a significant regression line describing the relationship between Log-Log transformed Protura density and $\mathrm{pH}$ values $\left[\mathrm{a}=-10.4, \mathrm{~b}=10.7, \mathrm{r}^{2}=0.7328\right.$, $\mathrm{p}$ (uncorr) $=$ $0.03]$.

\section{Analysis of ecological distribution}

Species assemblages from different macro-habitats were generally statistically different from each other, although we were unable to detect a close relationship between a single species and a particular habitat. One-way ANOSIM, both using the Bray-Curtis or Jaccard similarity index, gave significant 'p' values for the majority of macro-habitat couplets for genus and species level data. The clustering of macro-habitats based on genera assemblages is shown in Figure 2: bootstrap values show strong support for all the nodes but two.

SIMPER analysis was performed between each pair of macro-habitats for which Protura assemblages differed indicating which genera could be considered responsible for these differences. These results, together with those in Table 4 showing the distribution of the species in the macrohabitats, allowed us to outline some ecological considerations (Q4).

\section{Table 4}

Species distribution of Protura in Italy and Corsica. The abundance in the geographical units is shown for the whole dataset of 5910 specimens identified at species level from 499 sampling 
localities in the study area, including also, for greater completeness, those for which data about habitat and elevation were missing. Habitat legend: 1 = Acacia trees, 2 = Alder (Alnus sp.) forest, 3 = Beech (Fagus sylvatica) forest, $4=$ bushes, 5 = cave, $6=$ Chestnut (Castanea sativa) forest, $7=$ city park, 8 = coniferous forest, $9=$ Cork oak (Quercus suber) forest, $10=$ Downy oak (Quercus pubescens) forest, 11 = Fir (Abies alba) forest, 12 = Hazelnuts (Corylus avellana), $13=$ Holm oak (Quercus ilex) forest, 14 = Hornbeam (Carpinus betulus) forest, $15=$ Larch (Larix decidua) forest, 16 = Locust tree (Robinia pseudoacacia) forest, 17 = Manna ash (Fraxinus ornus) forest, $18=$ Maple (Acer sp.) trees, $19=$ meadow, $20=$ garden, $21=$ Mediterranean maquis, $22=$ mixed forest, 23 = mole nest, 24 = mosses, $25=$ near water, 26 = Oak (Quercus spp.) forest, $27=$ Olive (Olea europaea) trees, 28 = Pine (Pinus spp.) forest, $29=$ ravine, $30=$ reeds near seashore, $31=$ Sessile oak (Quercus petraea) forest, $32=$ under stones, $33=$ under stones near water, $34=$ Vineyard, $35=$ Willow (Salix sp.) forest. Macrohabitat legend: $\mathrm{A}=$ Mediterranean maquis, $\mathrm{B}=$ Mediterranean sclerophyllous forest, $\mathrm{C}=$ basal plane deciduous forest, $\mathrm{D}=$ sub-montane deciduous forest, $\mathrm{E}=$ montane deciduous forest, $\mathrm{F}=$ montane coniferous forest, $\mathrm{G}=$ olive trees, $\mathrm{H}=$ vineyards. Maximal recorded elevation is reported in the last column.

\begin{tabular}{|c|c|c|c|c|c|c|c|c|c|c|c|c|c|c|c|}
\hline & $\frac{n}{z}$ & 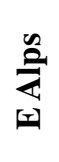 & $\begin{array}{l}\frac{n}{2} \\
\frac{2}{2} \\
\frac{3}{2}\end{array}$ & 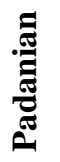 & $\frac{\bar{\partial}}{4}$ & 迹 & 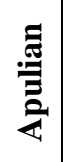 & 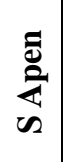 & 馬 & 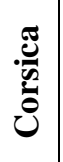 & 爫 & 䲶 & 苛 & 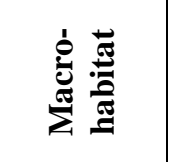 & 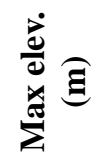 \\
\hline Ionescuellum condei & 0 & 1 & 6 & 0 & 2 & 0 & 0 & 0 & 0 & 0 & 0 & 0 & 19,27 & $\mathrm{G}$ & 2000 \\
\hline Protentomon berlesei & 0 & 1 & 0 & 1 & 0 & 0 & 0 & 0 & 0 & 0 & 0 & 0 & & & \\
\hline Protentomon perpusillum & 0 & 0 & 0 & 0 & 1 & 0 & 0 & 0 & 0 & 0 & 0 & 0 & 22 & $\mathrm{E}$ & \\
\hline Proturentomon condei & 5 & 0 & 0 & 0 & 0 & 1 & 0 & 0 & 0 & 0 & 0 & 0 & 26,32 & $\mathrm{D}$ & 780 \\
\hline Proturentomon discretum & 0 & 0 & 0 & 0 & 0 & 0 & 0 & 0 & 0 & 2 & 0 & 0 & 20 & 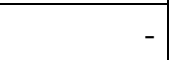 & 28 \\
\hline Proturentomon minimum & 1 & 0 & 2 & 10 & 5 & 3 & 0 & 0 & 0 & 0 & 7 & 0 & $\begin{array}{r}7,9,13 \\
21,26, \\
27,28,34 \\
\end{array}$ & $\begin{array}{r}\mathrm{A}, \mathrm{B}, \mathrm{C}, \\
\mathrm{G}, \mathrm{H}\end{array}$ & 800 \\
\hline Proturentomon noseki & 0 & 0 & 0 & 2 & 0 & 0 & 0 & 0 & 0 & 0 & 0 & 0 & 34 & $\mathrm{H}$ & \\
\hline Proturentomon pilosum & 0 & 0 & 0 & 1 & 1 & 0 & 0 & 0 & 0 & 0 & 0 & 0 & - & - & - \\
\hline Proturentomon pectinatum & 0 & 0 & 0 & 0 & 0 & 0 & 0 & 0 & 0 & 2 & 0 & 0 & 29 & - & 250 \\
\hline Acerentulus apuliacus & 0 & 0 & 0 & 0 & 0 & 0 & 16 & 32 & 0 & 0 & 0 & 0 & $3,18,22$ & $\mathrm{C}, \mathrm{D}$ & 600 \\
\hline Acerentulus condei & 0 & 0 & 0 & 0 & 0 & 0 & 0 & 0 & 0 & 0 & 2 & 0 & 24 & 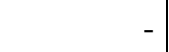 & - \\
\hline Acere & 1 & 9 & 123 & 76 & 236 & 3 & 1 & 1 & 0 & 4 & 0 & 0 & $\begin{array}{r}2,3,6,7 \\
9,10,12 \\
13,14 \\
16,20 \\
22,26 \\
28,29 \\
33,34 \\
\end{array}$ & $\begin{array}{r}\mathrm{B}, \mathrm{C}, \mathrm{D}, \\
\mathrm{E}, \mathrm{H}\end{array}$ & 1600 \\
\hline Acerentulus cunhai & 0 & 0 & 0 & 2 & 2 & 0 & 0 & 0 & 0 & 0 & 0 & 0 & - & -1 & 500 \\
\hline Acerentulus exiguus & 0 & 6 & 0 & 0 & 3 & 0 & 0 & 0 & 0 & 36 & 7 & 0 & $\begin{array}{r}6,12,13 \\
17,20 \\
22,28\end{array}$ & $\mathrm{~B}, \mathrm{C}, \mathrm{D}, \mathrm{E}$ & 1200 \\
\hline
\end{tabular}




\begin{tabular}{|c|c|c|c|c|c|c|c|c|c|c|c|c|c|c|c|}
\hline & $\sum_{z}^{\infty}$ & 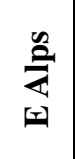 & $\begin{array}{l}\frac{n}{2} \\
\frac{2}{2} \\
\infty\end{array}$ & 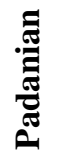 & 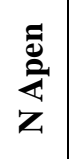 & 这 & 吾 & 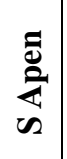 & 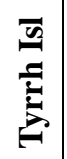 & 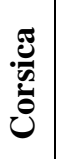 & 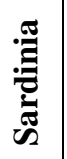 & $\begin{array}{l}\frac{2}{0} \\
\frac{0}{2}\end{array}$ & 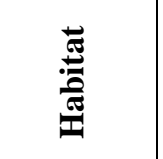 & 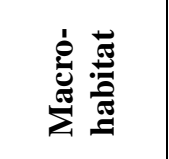 & 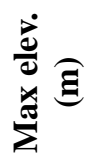 \\
\hline & & & & & & & & & & & & & $29,32,33$ & & \\
\hline Acerentulus gisini & 3 & 0 & 0 & 1 & 1 & 0 & 0 & 0 & 0 & 0 & 0 & 0 & 6 & $\mathrm{D}$ & 780 \\
\hline Acerentulus shrubovychae & 0 & 0 & 30 & 0 & 0 & 0 & 0 & 0 & 0 & 0 & 0 & 0 & 22 & $\mathrm{E}$ & 1165 \\
\hline Acerentulus terricola & 0 & 0 & 3 & 0 & 0 & 0 & 0 & 0 & 0 & 0 & 0 & 0 & 9 & $\mathrm{~B}$ & 100 \\
\hline Acerentulus traegardhi & 56 & 0 & 0 & 0 & 10 & 4 & 1 & 0 & 0 & 0 & 0 & 0 & $6,26,27$ & $\mathrm{C}, \mathrm{D}, \mathrm{E}, \mathrm{G}$ & 970 \\
\hline Acerentulus tuxeni & 0 & 6 & 0 & 0 & 0 & 0 & 0 & 0 & 0 & 0 & 0 & 0 & 1 & $\mathrm{C}$ & 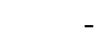 \\
\hline Gracilentulus corsicanus & 0 & 0 & 0 & 0 & 0 & 0 & 0 & 0 & 0 & 39 & 0 & 0 & 5 & - & 600 \\
\hline Gracilentulus gracilis & 1 & 0 & 3 & 1 & 9 & 0 & 0 & 0 & 0 & 3 & 4 & 0 & $\begin{array}{c}7,8,9, \\
13,14, \\
20,26\end{array}$ & $\mathrm{~B}, \mathrm{D}, \mathrm{F}$ & 1320 \\
\hline Gracilentulus meridianus & 0 & 0 & 0 & 0 & 0 & 0 & 0 & 0 & 0 & 2 & 9 & 0 & 13 & B & 850 \\
\hline Gracilentulus orousseti & 0 & 0 & 0 & 0 & 0 & 0 & 0 & 0 & 0 & 7 & 0 & 0 & 5 & - & 600 \\
\hline Gracilentulus sardinianus & 0 & 0 & 0 & 0 & 0 & 0 & 0 & 0 & 0 & 0 & 9 & 0 & 13,26 & $\mathrm{~B}, \mathrm{D}$ & 700 \\
\hline Podolinella ruseki & 0 & 0 & 0 & 0 & 29 & 0 & 0 & 0 & 0 & 0 & 0 & 0 & 13,34 & $\mathrm{~B}, \mathrm{H}$ & 100 \\
\hline Acerentomon affine & 11 & 12 & 7 & 17 & 99 & 0 & 0 & 0 & 0 & 1 & 0 & 0 & $\begin{array}{r}3,6,9 \\
10,13 \\
14,16, \\
17,22,27\end{array}$ & $\begin{array}{r}\mathrm{B}, \mathrm{C}, \mathrm{D}, \\
\mathrm{E}, \mathrm{G}\end{array}$ & 2000 \\
\hline Acerentomon balcanicum & 0 & 7 & 0 & 0 & 33 & 9 & 4 & 13 & 0 & 0 & 4 & 0 & $\begin{array}{r}1,3,19 \\
21,22 \\
26,27,32\end{array}$ & $\begin{array}{r}\mathrm{A}, \mathrm{C}, \mathrm{D}, \\
\mathrm{E}, \mathrm{G}\end{array}$ & 1600 \\
\hline Acerentomon baldense & 0 & 13 & 0 & 0 & 0 & 0 & 0 & 0 & 0 & 0 & 0 & 0 & 3 & $\mathrm{E}$ & $1400 *$ \\
\hline Acerentomon condei & 0 & 0 & 0 & 0 & 0 & 0 & 0 & 0 & 0 & 0 & 10 & 0 & 13 & B & 480 \\
\hline Acerentomon doderoi & 9 & 0 & 191 & 0 & 12 & 0 & 0 & 0 & 0 & 0 & 0 & 0 & $\begin{array}{r}3,5,6,9 \\
10,13 \\
14,21,22 \\
\end{array}$ & $\begin{array}{r}\mathrm{A}, \mathrm{B}, \mathrm{C}, \\
\mathrm{D}, \mathrm{E}\end{array}$ & 1350 \\
\hline Acerentomon gallicum & 0 & 0 & 143 & 2 & 0 & 0 & 0 & 0 & 0 & 0 & 0 & 0 & $\begin{array}{r}3,5,13, \\
22,24\end{array}$ & $\mathrm{~B}, \mathrm{D}, \mathrm{E}$ & 1690 \\
\hline Acerentomon italicum & 2 & 183 & 350 & 34 & 762 & 1 & 0 & 0 & 16 & 8 & 0 & 0 & $\begin{array}{r}1,3,6,7 \\
9,10,12 \\
13,14 \\
16,17 \\
21,22 \\
26,28,33 \\
\end{array}$ & $\begin{array}{r}\mathrm{A}, \mathrm{B}, \mathrm{C}, \\
\mathrm{D}, \mathrm{E}\end{array}$ & 2000 \\
\hline Acerentomon maius & 29 & 292 & 571 & 71 & 226 & 10 & 0 & 0 & 2 & 0 & 0 & 0 & $\begin{array}{r}3,6,7,9 \\
10,13 \\
14,16 \\
22,24 \\
26,31,35 \\
\end{array}$ & $\mathrm{~B}, \mathrm{C}, \mathrm{D}, \mathrm{E}$ & 1690 \\
\hline Acerentomon meridionale & 0 & 0 & 0 & 43 & 10 & 83 & 4 & 2 & 53 & 3 & 0 & 18 & $\begin{array}{r}3,7,13 \\
21,26 \\
27,32\end{array}$ & $\begin{array}{c}\mathrm{A}, \mathrm{B}, \mathrm{C}, \\
\mathrm{D}, \mathrm{E}, \mathrm{G}\end{array}$ & 1600 \\
\hline Acerentomon microrhinus & 24 & 50 & 5 & 105 & 218 & 1 & 0 & 1 & 0 & 0 & 0 & 0 & $\begin{array}{r}3,4,6,7 \\
10,13 \\
14,16 \\
19,22 \\
23,24 \\
26,27 \\
\end{array}$ & $\begin{array}{r}\mathrm{B}, \mathrm{C}, \mathrm{D}, \\
\mathrm{E}, \mathrm{G}\end{array}$ & 1000 \\
\hline Acerentomon noseki & 2 & 0 & 0 & 0 & 0 & 0 & 0 & 0 & 0 & 0 & 0 & 0 & 3 & $\mathrm{E}$ & 1100 \\
\hline Acerella mиscorum & 0 & 142 & 21 & 1 & 0 & 7 & 0 & 0 & 0 & 0 & 0 & 0 & $\begin{array}{r}3,6,12, \\
13\end{array}$ & $\mathrm{~B}, \mathrm{D}, \mathrm{E}$ & 1360 \\
\hline
\end{tabular}




\begin{tabular}{|c|c|c|c|c|c|c|c|c|c|c|c|c|c|c|c|}
\hline & $\frac{n}{2}$ & 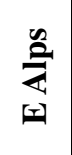 & $\sum_{\infty}^{\infty}$ & 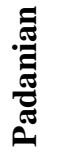 & $\begin{array}{l}\overline{0} \\
Z^{2} \\
\mathbf{Z}\end{array}$ & $\sum_{u}^{\frac{\pi}{2}}$ & 金 & 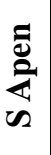 & है & ن⿺辶ِّ & : & हैं & $\frac{\vec{\pi}}{\frac{\pi}{0}}$ & 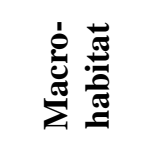 & 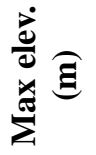 \\
\hline Acerella tiarnea & 9 & 63 & 89 & 0 & 80 & 2 & 0 & 2 & 0 & 1 & 0 & 0 & $\begin{array}{r}1,2,3,4, \\
9,12,13, \\
14,17, \\
19,21, \\
22,26\end{array}$ & $\begin{array}{r}\mathrm{A}, \mathrm{B}, \mathrm{C}, \\
\mathrm{D}, \mathrm{E}, \mathrm{G}\end{array}$ & 1900 \\
\hline Isoentomon atlanticum & 0 & 0 & 0 & 0 & 0 & 0 & 0 & 0 & 0 & 4 & 0 & 0 & 20,25 & - & 40 \\
\hline Eosentomon armatum & 0 & 4 & 0 & 1 & 0 & 0 & 0 & 0 & 0 & 0 & 0 & 12 & 1,13 & $\mathrm{~B}, \mathrm{C}$ & - \\
\hline Eosentomon delicatum & 0 & 7 & 1 & 23 & 11 & 0 & 0 & 0 & 0 & 59 & 0 & 0 & $\begin{array}{r}9,13,16, \\
17,19, \\
20,22, \\
24,25, \\
29,32,33 \\
\end{array}$ & $\mathrm{~B}, \mathrm{C}, \mathrm{E}$ & 1100 \\
\hline Eosentomon foroiuliense & 0 & 1 & 0 & 0 & 0 & 0 & 0 & 0 & 0 & 0 & 0 & 0 & 3 & $\mathrm{E}$ & 1200 \\
\hline Eosentomon noseki & 2 & 10 & 14 & 4 & 92 & 7 & 0 & 0 & 0 & 0 & 0 & 0 & $\begin{array}{r}1,2,3,6, \\
9,10,13, \\
14,15, \\
19,21 \\
26,27 \\
32,34 \\
\end{array}$ & $\begin{array}{r}\mathrm{A}, \mathrm{B}, \mathrm{C}, \\
\mathrm{D}, \mathrm{E}, \mathrm{F}, \mathrm{G}, \\
\mathrm{H}\end{array}$ & 1800 \\
\hline Eosentomon romanum & 0 & 0 & 0 & 3 & 0 & 5 & 0 & 0 & 0 & 0 & 0 & 0 & - & - & - \\
\hline Eosentomon transitorium & 27 & 105 & 134 & 68 & 18 & 82 & 0 & 0 & 0 & 69 & 109 & 0 & $\begin{array}{r}3,6,8,9, \\
10,11,12, \\
13,14, \\
15,16, \\
18,19, \\
20,21, \\
22,24, \\
25,28, \\
29,31, \\
32,33\end{array}$ & $\begin{array}{r}\mathrm{A}, \mathrm{B}, \mathrm{C}, \\
\mathrm{D}, \mathrm{E}, \mathrm{F}\end{array}$ & 2000 \\
\hline
\end{tabular}

* In the original description (TORTI, C., 1986. A new Protura species from Italy Acerentomon baldense sp. n. (Protura, Acerentomidae). Revue suisse de Zoologie, 93, 63-65) quota (400 m elev.) was wrong.

Vineyards and montane coniferous forests were (see also clustering in Fig. 2) the most differentiated macro-habitats. Vineyards were characterized by the dominance of the genera Proturentomon and Podolinella, which were scarce or absent in other habitats, and by the absence of Acerentomon, elsewhere the most abundant. Montane coniferous forests were dominated by Eosentomon: 54 out of 55 specimens collected were E. transitorium. Mediterranean maquis and olive tree plantations shared a similar proportion of Acerentomon and Acerella specimens; in the olive tree plantations the dominant species were Acerentomon affine and Acerentomon microrhinus, while in Mediterranean maquis A. italicum was the most abundant species. Montane deciduous and basal deciduous forests were characterized by the dominance of Acerentomon and Acerentulus, and by the absence of Gracilentulus. The most abundant species in montane deciduous forests were $A$. maius, A. doderoi and A. confinis; A. italicum, A. microrhinus, A. confinis and A. maius dominated in the basal deciduous forests. Acerentomon, Acerentulus and Eosentomon (especially E. noseki) 
were the most represented in the submontane deciduous forests. Mediterranean sclerophyllous forests were dominated overall by Acerentomon (A. italicum and A. maius mainly), followed by Eosentomon (E. transitorium).

In total, 13 collections occurred in urban areas (city parks and urbanized environments): 11 in Genoa, one in Arenzano (a small town near Genoa) and one in Florence. Among the 109 Protura specimens collected and identified to species level, 57 were A. italicum, 37 A. microrhinus, and five A. confinis. These are among the most common species in Italy. Other species identified were Ionescuellum condei, Acerentulus exiguus, Gracilentulus gracilis, A. maius, Acerentomon meridionale, Eosentomon delicatum and E. noseki.

The number of species identified in each locality ranged from one to 11 (in the cork oak wood in Bergeggi - Galli et al., 2012), while the number of species for each collecting session was $1.81 \pm$ 1.16 (range: $1-8)$.

We observed a clear pattern in the width of ecological distribution in the four geographical macrounits (Q5). North Italy and islands were inhabited both by widespread euryoecious species, and by those colonizing a more limited range of habitats with a narrow geographical distribution in Italy. Moving through the center to the south of Italy, Protura assemblages included proportionally evermore widespread species both from a geographical and an ecological point of view. However, this trend was not statistically significant when analysed using the Kruskal-Wallis test.

The geographical spread of species (in terms of the number of geographical units for each species) displayed a linear relationship with the ecological spread (number of habitats where each species was recorded). The slope of the regression line was 0.506 (zero intercept $-r^{2}=0.776, p$ (uncorr) $<<$ $0.01)$.

Unfortunately, we do not have samples collected from the Alpine plain at elevations above $2000 \mathrm{~m}$, and few collections were taken from the montane plain. We observed that 11 species were collected above $1500 \mathrm{~m}$ (Table 4), and that nine of the species had wide geographical distributions; especially A. confinis and E. transitorium.

\section{Sex ratio and phenology}

The number of males and females collected and the relative sex ratio for each dominant and subdominant species are shown in Table 5 (Q6). Values for 12 species of Acerentomata differed significantly from unity; in contrast, the sex ratio for all the Eosentomata species were balanced.

\section{Table 5}

Sex ratio calculated for dominant and sub-dominant species of Protura in Italy. $\widehat{\jmath} \hat{\sigma}=$ number of males, $q Q+=$ number of females, sex ratio $\widehat{\partial} \hat{\partial} / Q q+, \chi^{2}$ and relative $\mathrm{p}$ values for balanced sex ratio. 


\begin{tabular}{|c|c|c|c|c|c|}
\hline Species & $\hat{O} \sigma^{\lambda}$ & 우우 & Sex ratio & $\chi^{2}(1$ d.f $)$ & $\mathbf{p}$ \\
\hline Proturentomon minimum & 0 & 20 & 0 & 20 & $<<0.01$ \\
\hline Acerentulus apuliacus & 4 & 20 & 0.20 & 10.667 & $<0.01$ \\
\hline Acerentulus confinis & 147 & 229 & 0.64 & 17.883 & $<<0.01$ \\
\hline Acerentulus exiguus & 9 & 13 & 0.69 & 0.72727 & 0.70 \\
\hline Acerentulus gisini & 4 & 10 & 0.40 & 2.5714 & 0.28 \\
\hline Acerentulus shrubovychae & 5 & 17 & 0.29 & 6.5455 & $<0.05$ \\
\hline Acerentulus traegardhi & 15 & 14 & 1.07 & 0.034483 & 0.98 \\
\hline Acerentomon affine & 63 & 75 & 0.84 & 1.0435 & 0.59 \\
\hline Acerentomon balcanicum & 25 & 31 & 0.81 & 0.64286 & 0.72511 \\
\hline Acerentomon doderoi & 84 & 112 & 0.75 & 4 & $<0.05$ \\
\hline Acerentomon gallicum & 64 & 135 & 0.47 & 25.332 & $<<0.01$ \\
\hline Acerentomon italicum & 527 & 686 & 0.77 & 20.866 & $<<0.01$ \\
\hline Acerentomon maius & 371 & 469 & 0.79 & 11.433 & $<<0.01$ \\
\hline Acerentomon meridionale & 67 & 122 & 0.55 & 16.005 & $<<0.01$ \\
\hline Acerentomon microrhinus & 119 & 186 & 0.64 & 14.718 & $<<0.01$ \\
\hline Acerella muscorum & 5 & 28 & 0.18 & 16.03 & $<<0.01$ \\
\hline Acerella tiarnea & 59 & 155 & 0.38 & 43.065 & $<<0.01$ \\
\hline Eosentomon delicatum & 39 & 29 & 1.34 & 1.4706 & 0.48 \\
\hline Eosentomon noseki & 50 & 49 & 1.02 & 0.010101 & 0.99 \\
\hline Eosentomon transitorium & 204 & 192 & 1.06 & 0.36364 & 0.83 \\
\hline
\end{tabular}

The analysis of phenology (Q7) was undertaken for the dominant species, based on the rather low number of juveniles (from larva I to maturus junior, or pre-imago for Acerentomidae) (Fig. 3B-F). Acerentomon gallicum and A. maius presented a pattern with two peaks, both of adults and juveniles, during the spring-summer and mid-autumn period. In A. italicum, juveniles were detected every month with a peak in July. In A. microrhinus juveniles occurred only as a single peak during late summer-early autumn. Finally, Eosentomon transitorium showed two maxima of juveniles in March, and from June to August (adults showing a third peak in October, when no juveniles were collected).

\section{Discussion}

Protura are quite common but never as abundant as other soil arthropods such as oribatid mites and springtails. The density values we recorded in Ligurian soils (NW-Italy) are comparable with those already known in the European literature, where values from few hundreds up to $16000 / \mathrm{m}^{2}$ were found (Axelsson et al., 1973; Huhta \& Koskenniemi, 1975; Nosek, 1975; Gunnarsson, 1980; Stumpp, 1990; Sterzyńska et al., 2012). Higher densities of Protura have been observed in coniferous forests (Gunnarsson 1980). In our dataset the number of samples collected in coniferous 
forests (moreover not natural) and analysed quantitatively was too low to verify this observation and further sampling is required.

The aggregated distribution of Protura evidenced in this study was observed previously by Raw (1956), Walker and Rust (1975) and Gunnarsson (1980). The aggregation of populations in the field is typical for this taxon, and is possibly due to the gathering of individuals around fungal food sources. This kind of distribution is quite common among soil arthropods (cf. Wardle, 2002) and is due to environmental pressures or to the production of aggregation pheromones. Several species of Collembola show aggregated distributions and are known to produce aggregation pheromones for sexual attraction but also to promote grouping at rich food sources; close aggregation may also allow springtails to create their own microclimate and prevent desiccation (cf. Hopkin, 1997).

Our analysis of soil samples from chestnut and beech forests on Mt Antola, Mt Penna and Mt Zatta (Genoa province) suggests that vegetation and the physico-chemical characteristics of the soils on different geological substrates may influence Proturan density. The number of Protura was indeed different between the two habitats, and between marly limestone soils (slightly alkaline, with 0.01 $\mathrm{mm}$ grain size) on one side and flysh and siltstones/sandstones soils (slightly/moderately acidic, with $0.05 \mathrm{~mm}$ grain size) on the other. The apparent influence of soil $\mathrm{pH}$ on Proturan densities we detected cannot be generalized. Minor (2008), studying New Zealand forests, was unable to find any relationship between soil organic matter, nitrogen, phosphorus, $\mathrm{pH}$, cation exchange capacity, and the density of Protura. Such an absence of direct correlation between abundance of soil microarthropods and soil chemistry seems to be common (Laiho et al. 2001). On the other hand, da Silva et al. (2016) detected a negative correlation of $\mathrm{pH}$ with Collembola richness, identifying it as the main soil parameter influencing springtail communities. Furthermore, Maraun and Scheu (2000) showed an indirect influence of $\mathrm{pH}$ on oribatid mites density.

Proturan assemblages typical of the main macro-habitats we analysed conform to the observations of Nosek (1973, 1975), who noted that European Protura form relatively stable community associations in different types of forests, presumably due to species having similar environmental requirements. In New Zealand, Minor (2008) observed that Proturan assemblage composition was significantly related to forest type, and she deduced that their abundance patterns may reflect the association with fungal communities in the soil.

To date we have collected only a few samples from a limited number of urban areas, so we are not able to indicate if some species are more tolerant of urbanization. However, we do know that the dominant species of Protura that we found in urban habitats (A. italicum, A. microrhinus and A. confinis) are among the most common and widespread (therefore the most adaptable) species in Italy. In Luxemburg Szeptycki et al. (2003) found a very specific fauna in town parks, gardens and other anthropogenic habitats. Some species (Acerentulus cunhai, G. gracilis, Proturentomon 
discretum, Berberentulus polonicus and Eosentomon luxembourgense) were much more common or only present in these habitat types; others (Acerentomon nemorale, A. brevisetosum, Acerella remyi, Eosentomon silesiacum and E. stompi) were absent from anthropogenic habitats. Christian and Szeptycki (2004) described a species distribution along an urban gradient in Vienna and found that some Protura (E. luxemburgense and E. mirabile) seemed to be particularly well adapted to anthropization. Imadate and Ohnishi (1993) and Nakamura (2014) noted that the Japanese Protura comprise two major stocks. Eosentomon sakura, Paranisentomon tuxeni and Eosentomon tokiokai, members of the southern stock, are more tolerant to the deterioration of the environment and recent urbanization. In contrast, Eosentomon asahi, belonging to the northern stock, mainly occurs in deciduous broadleaved forests and plantations and is less abundant in urban areas, presumably due to its sensitivity to the degradation of natural environment.

The species richness we recorded for each sampling locality seems to agree with the range of 1-10 Protura species/site observed in other countries (Stumpp, 1990; Blesic, 2005; Sterzyńska et al., 2012). The maximum number of syntopic species known to date is 23 in a Downy Oak stand (Quercus pubescens) over platy marl (390 m elev.) in the Wienerwald (Christian and Szeptycki, 2004).

The relationship between the ecological adaptability of species and the size of their geographical range was already documented by Nosek (1975) for the European species. He observed that there are species (e.g. E. transitorium and Proturentomon minimum) that cover a wide range and can be regarded as ecologically tolerant of a broad range of abiotic and biotic factors, whereas other species are ecologically intolerant and confined to smaller ranges and often restricted to natural habitats characterized by luxuriant vegetation.

Even though we have few collections made at higher elevations (none over $2000 \mathrm{~m}$ in the Alpine plane) we have observed the association between geographical range amplitude and elevation reached by Protura species on mountains in Italy, which has also been noticed by Shrubovych and Sterzyńska (2017) in the Ukraine. It seems likely that Protura occurring at higher elevations can tolerate a wider range of climatic conditions (according to the Rapoport's altitudinal rule) and so they also have generally wider distributions.

We detected an unbalanced sex ratio in favour of females in 12 dominant species of Acerentomata but not in the three dominant Eosentomon species (Table 5). Gunnarsson (1980), however, in an oak wood in Sweden recorded that females of Eosentomon germanicum were more than twice as numerous as the males. It can therefore be assumed to be a common feature among Proturan populations probably due to the capability of a single male to fertilize more females for example through spermatophores. Protura are known to be characterized by a great sperm variability, exhibiting both flagellated and aflagellated immotile sperm, but it is still unknown if the sperm 
transfer is direct or indirect through spermatophores (Dallai et al., 2010a, b). The sperm structure of many species and the unbalanced sex ratio could support the latter hypothesis. Inter alia, $P$. minimum could be a parthenogenetic species: there is only a single male known in literature (collected in Bosnia and Hercegovina in 1968 - Nosek, 1973), and its identification could be wrong. Parthenogenesis in Protura has never been confirmed experimentally, but Minor (2008) detected four species in New Zealand whose populations were composed of only females and the absence or rarity of males in the Australian and New Zealand populations of some Protura species has been previously highlighted by Tuxen (1967, 1985). Another example is Andinentulus rapoporti. Shrubovych et al. (2014) and Galli (unpubl.) examined many specimens of this South-American species, finding only females.

Scarcity of records of juveniles - already highlighted by Walker and Rust (1975) - makes it difficult to identify well defined phenologies of the majority of species. We were able to describe clear patterns only for five dominant species (Fig. 3), which parallel those found by Imadate (1974) for some Japanese Protura. Phenology, however, probably varies among different habitats, geographic units [see the case of A. italicum in Galli et al. (2016)] and elevations. For example, Balkenhol (1994) in Germany described a three-peaked pattern for A. gallicum that was different from the double-peaked phenology we described for the same species in Italy (Fig 3B). Furthermore, in Serbia, Mitrovski Bogdanović and Blesic (2011) described a double-peaked phenology (September and June-July) for E. transitorium, which is quite different from what we outlined for this species in Italy (Fig. 3F).

\section{Conclusions}

Protura still remains one of the least known taxon from an ecological point of view. Our consideration of almost all the known literature about their ecology, and the elaboration of our data on the Italian fauna, has barely allowed us to define a generic framework concerning their ecological distribution. Moreover, the lack or scarcity of data for many central and southern Italian regions did not allow us to develop patterns about the geographical distribution of Protura in Italy in relation to their ecology. Only further targeted sampling efforts can address the shortfall in data, especially in those areas for which information is lacking or too scarce.

Italy constitutes a "laboratory" offering unlimited possibilities for further research as many different kinds of habitats and geological formations are distributed along a quite wide latitudinal and altitudinal range.

Much work remains to be done, and this paper should be considered a starting point for future research while not underestimating that other disciplines (e.g. pedology, mycology and botany) can also contribute towards a greater understanding of the ecology of these animals. 


\section{Acknowledgments}

We thank Dr Peter Schwendinger, Dr Roberto Poggi and Dr Leonardo Latella for information from the collections of Protura from the Geneva, Genoa and Verona Natural History Museums, respectively; Dr Maria Piera Rogantin for her help in the statistical analysis of data; Dr Giuseppina Barberis for her useful suggestions about habitat grouping; and Dr Birgit Balkenhol for her kind help in understanding some data from her $\mathrm{PhD}$ thesis, and for her useful suggestions. Many thanks also to the anonymous reviewers who helped us to significantly improve this paper.

\section{References}

Axelsson, B., Lohm, U., Lundkvist, H., Persson, T., Skoglund, J., Wirén, A., 1973. Effects of nitrogen fertilization on the abundance of soil fauna populations in a Scots pine stand. Stockholm, Royal College of Forestry Research Notes, No. 14.

Balkenhol, B., 1994. Die Besiedlung verinselter Waldflachen (Feldgeholze) mit Araneen, Carabiden und Proturen. PhD Thesis, Osnabröck University. 179 pp

Biondi, M., Urbani, F., D’Alessandro, P., 2013. Endemism patterns in the Italian leaf beetle fauna (Coleoptera, Chrysomelidae). ZooKeys, 332, 177-205. Doi: 10.3897/zookeys.332.5339

Blesic, B.F., 2005. Further contribution to the knowledge of Protura on Mountain Rudnik (Serbia). Kragujevac J. Sci., 27, 157-162.

Christian, E., Szeptycki, A., 2004. Distribution of Protura along an urban gradient in Vienna. Pedobiologia, 48, 445-452.

Condé, B., 1980. Nouvelle récoltes de Protures en Corse. Rev. Ecol. Biol. Sol, 17 (2), 295-301.

Da Silva, P.M., Carvalho, F., Dirilgen, T., Stone, D., Cramer, R., Bolger, T., Sousa, J.P., 2016. Traits of collembolan life-form indicate land use types and soil properties across an European transect. Appl. Soil. Ecol., 97, 66-77. doi: 10.1016/j.apsoil.2015.07.018

Dallai, R., Mercati, D., Bu, Y., Yin, Y.W., 2010a. Spermatogenesis and sperm structure of Acerella muscorum, (Ionescu, 1930) (Hexapoda, Protura). Tissue Cell., 42 (2), 97-104. doi: 10.1016/j.tice.2010.01.001

Dallai, R., Mercati, D., Bu, Y., Yin, Y.W., Callaini, G., Riparbelli, M.G., 2010b. The spermatogenesis and sperm structure of Acerentomon microrhinus (Protura, Hexapoda) with considerations on the phylogenetic position of the taxon. Zoomorphology, 129, 61-80. doi: 10.1007/s00435-009-0100-1

Galli, L., Bartel, D., Capurro, M., Pass, G., Sarà, A., Shrubovych, J., Szucsich, N., 2016. Redescription and review of the most abundant conehead in Italy: Acerentomon italicum Nosek, 1969 (Protura: Acerentomidae). Ital. J. Zool., 83 (1), 43-58. Doi: 10.1080/11250003.2015.1114686 
Galli, L., Capurro, M., Shrubovych, J., Torti, C., 2012. Phenology of Protura in a northwestern Italian forest soil (Hexapoda: Protura). Acta Zool. Cracov., vol. 55 (1), 33-43.

Galli, L., Capurro, M., Torti, C., 2011. Protura of Italy, with a key to species and their distribution. ZooKeys, 146, 19-67. Doi: 10.3897/zookeys.146.1885.

Galli, L., Zinni M., 2017. L'importanza dei Proturi nell'ambito delle biocenosi del suolo. Stato delle conoscenze. Atti Accademia Nazionale Italiana di Entomologia, LXV, 121-126.

Gunnarsson, B., 1980. Distribution, abundance and population structure of Protura in two woodland soils in southwestern Sweden. Pedobiologia 20, 254-262.

Hammer, Ø., Harper, D.A.T., Ryan P.D., 2001. PAST: Paleontological statistics software package for education and data analysis. Palaeontologia Electronica 4, 1-9. Available: http://palaeoelectronica.org/2001_1/past/issue1_01.htm. Accessed Oct 201621.

Hopkin, S.P., 1997. Biology of the Springtails (Insecta: Collembola). Oxford University Press, Oxford, UK. 330 pp.

Huhta, V., Koskenniemi, A., 1975. Numbers, biomass and community respiration of soil invertebrates in spruce forests at two latitudes in Finland. Ann. Zool. Fennici, 12, 164-182.

Imadaté, G., 1974. Protura (Insecta). Fauna japonica. Keigaku Publishing Co., Tokyo, 351 pp.

Imadaté, G., Ohnishi, J., 1993. Contributions towards a revision of the Protura fauna of Japan (VIII) Further collecting records from northern and eastern Japan. Bulletin of the Department of General Education, Tokyo Medical and Dental University, (23), 31-66.

Ives, A.R., 1991. Aggregation and coexistence in a carrion fly community. Ecological Monographs, 61, 75-94.

Laiho, R., Silvan, N., Carcamo, H., Vasander, H., 2001. Effects of water level and nutrients on spatial distribution of soil mesofauna in peatlands drained for forestry in Finland. Appl. Soil Ecol., $16,1-9$.

Maraun, M., Scheu, S., 2000. The structure of oribatid mites communities (Acari, Oribatida): patterns, mechanisms and implications for future research. Ecography, 23.3, 374-383.

Minor, M.A., 2008. Protura in native and exotic forests in the North Island of New Zealand. New Zeal. J. Zool., 35, 271-279.

Mitrovski Bogdanović, A., Blesic, B., 2011. Seasonal dynamics of Protura (Insecta) in an oak forest in Kragujevac (Serbia). Kragujevac J. Sci., 33, 77-82.

Nakamura, O., 2014. Habitat preference of species of the family Eosentomidae (Hexapoda: Protura) in Kanto district, central Japan. Bull. Saitama Mus. Nat. Hist. [N.S.], 8, 15-18.

Nosek, J., 1973. European Protura. Genève, Muséum d'Histoire Naturelle. 345 pp.

Nosek, J., 1975. Niches of Protura in Biogeocoenoses. Pedobiologia, 15, 290-298. 
Raw, F., 1956. The Abundance and Distribution of Protura in Grassland. J. Anim. Ecol., 25 (1), 1521.

Shrubovych., J., Schneider., C., D’Haese., C., 2014. Revision of genus Andinentulus (Protura Acerentomidae: Berberentulinae), with a key to South and Central American Acerentomidae species. Ann. Entomol. Soc. Am., 107 (3), 567-574. Doi: 10.1603/AN13151

Shrubovych, J., Sterzyńska, M., 2017. Diversity and distributional pattern of soil microarthropods (Protura) across a transitional zone in Ukraine. Can. Entomol., 149, 628-638. Doi: $10.4039 /$ tce. 2017.30

Sterzyńska, M., Orlov, O., Shrubovych, J., 2012. Effect of Hydrologic Disturbance Regimes on Protura Variability in a River Floodplain. Ann. Zool. Fennici, 49, 309-320. Doi: $10.5735 / 086.049 .0504$

Stumpp, J., 1990. Zur Ökologie einheimischer Proturen (Arthropoda: Insecta) in Fichtenforsten. Zoologische Beiträge, NF, 33, 345-432.

Szeptycki, A., 2007. Catalogue of the world Protura. Acta Zool. Cracov., 50B (1), 1-210.

Szeptycki, A., Stomp, N., Weiner, W.M., 2003. The Protura of Luxembourg. Ferrantia, 34, 5-49.

Tuxen, S.L., 1967. Australian Protura, their phylogeny and zoogeography. Z. Zool. Syst. Evol., 5, $1-53$.

Tuxen, S.L., 1985. Protura (Insecta). Fauna of New Zealand No. 9. Wellington, New Zealand, Department of Scientific and Industrial Research. 52 pp.

Walker, G.L., Rust, R.W., 1975. Seasonal distribution of Protura in three Delaware forests. Entomol. News, 86 (9-10), 187-198.

Wardle, D.A., 2002. Linking the Aboveground and Belowground Components. Monographs in Population Biology. Princeton University Press, Princeton, USA. 408 pp. 
Figure captions:

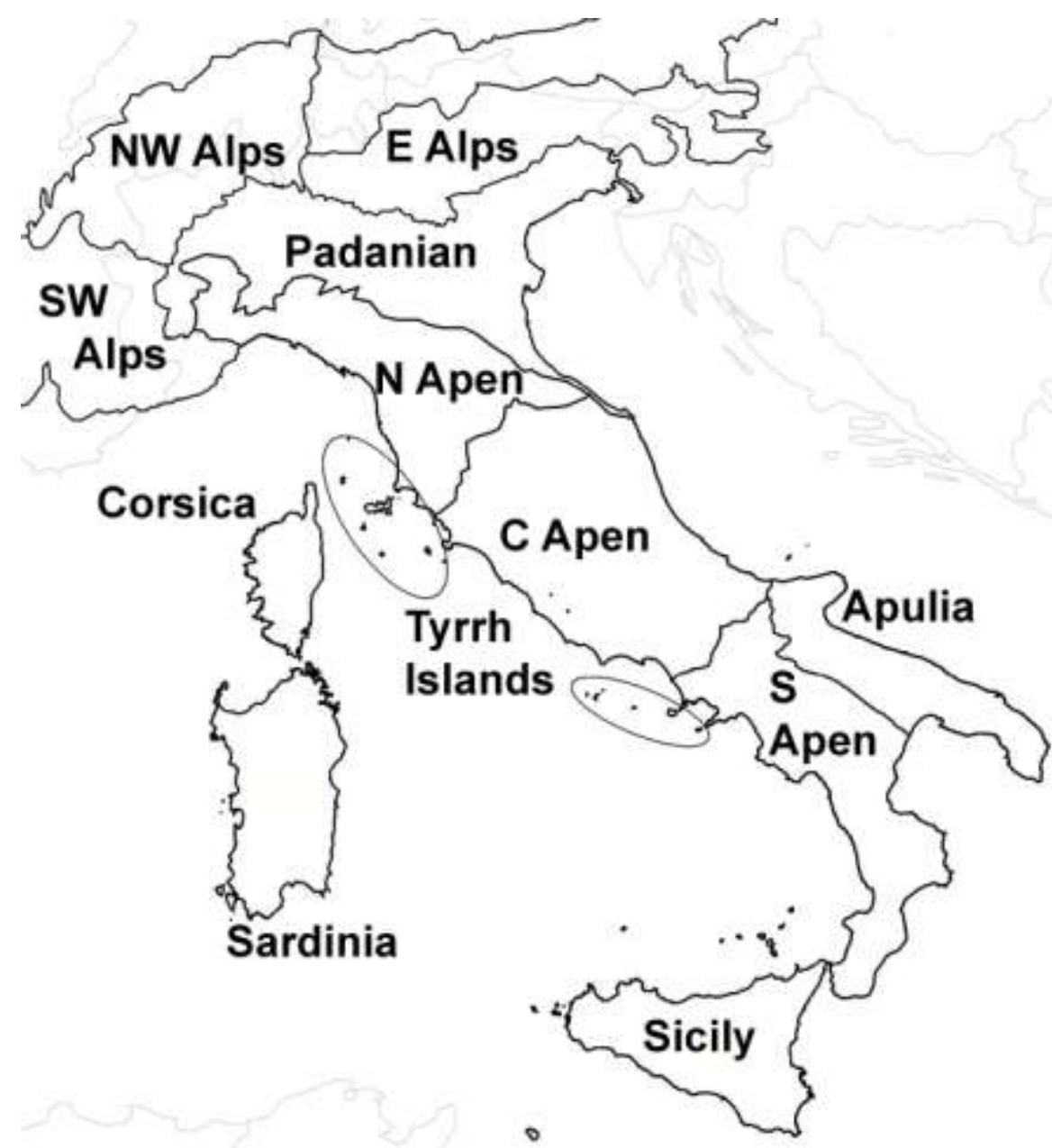

Fig. 1. Geographical units adopted for the analysis of Protura distribution in Italy and Corsica (modified from Biondi et al., 2013). 


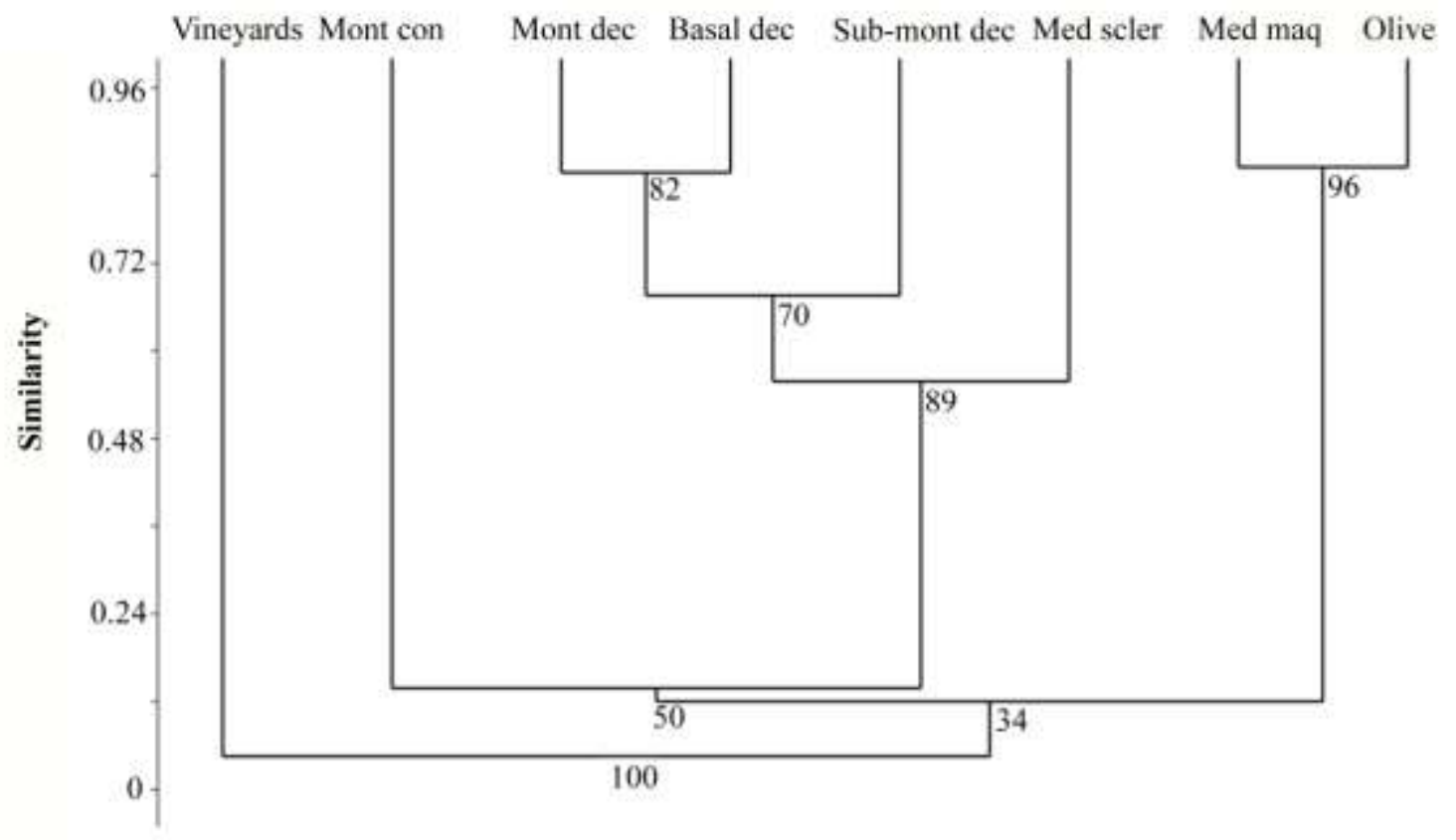

Fig. 2. Clustering of the macro-habitats based on the analysis of similarity of Protura genera assemblages: Paired group method, Bray-Curtis similarity index (1000 boots). 
A

B
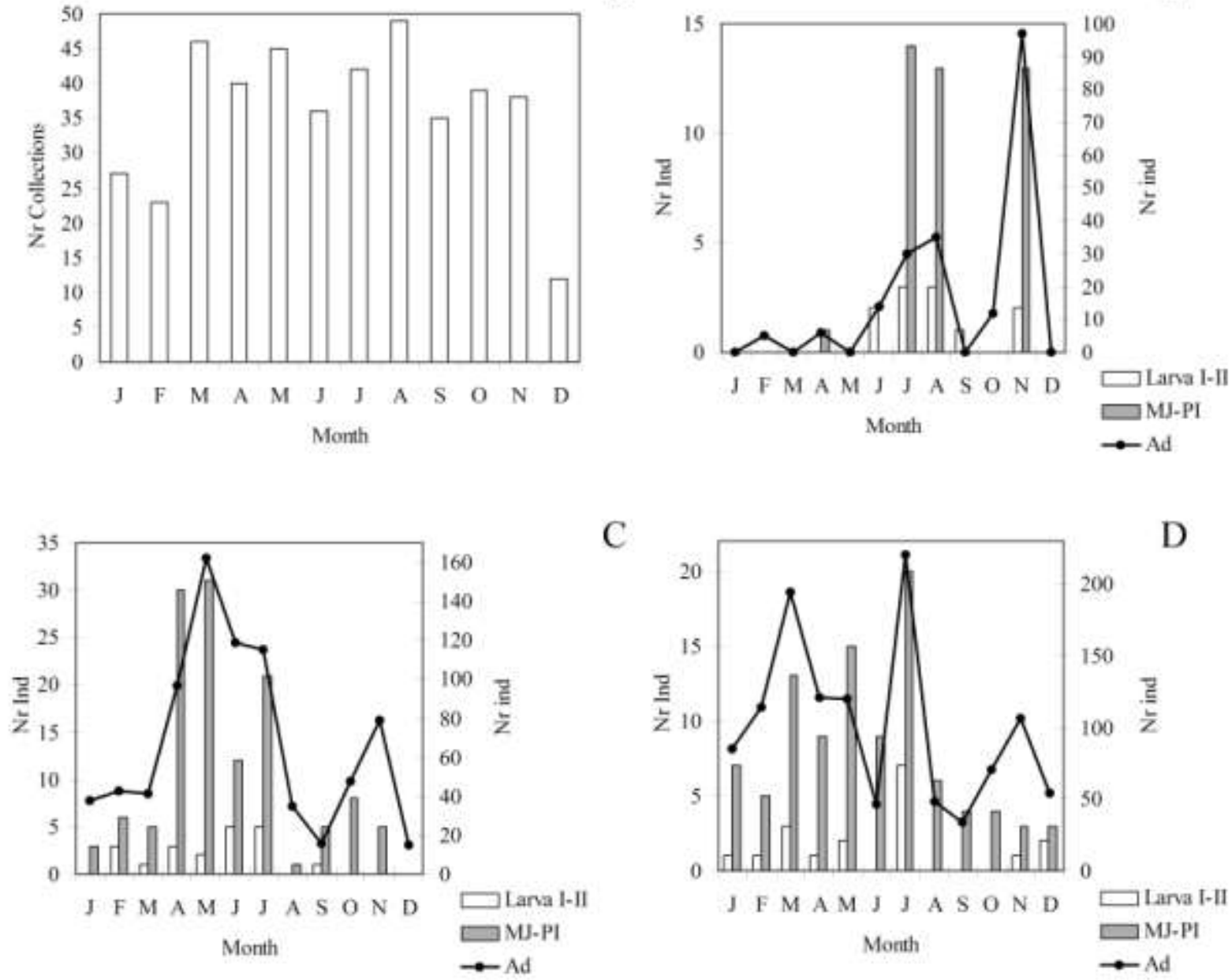

C
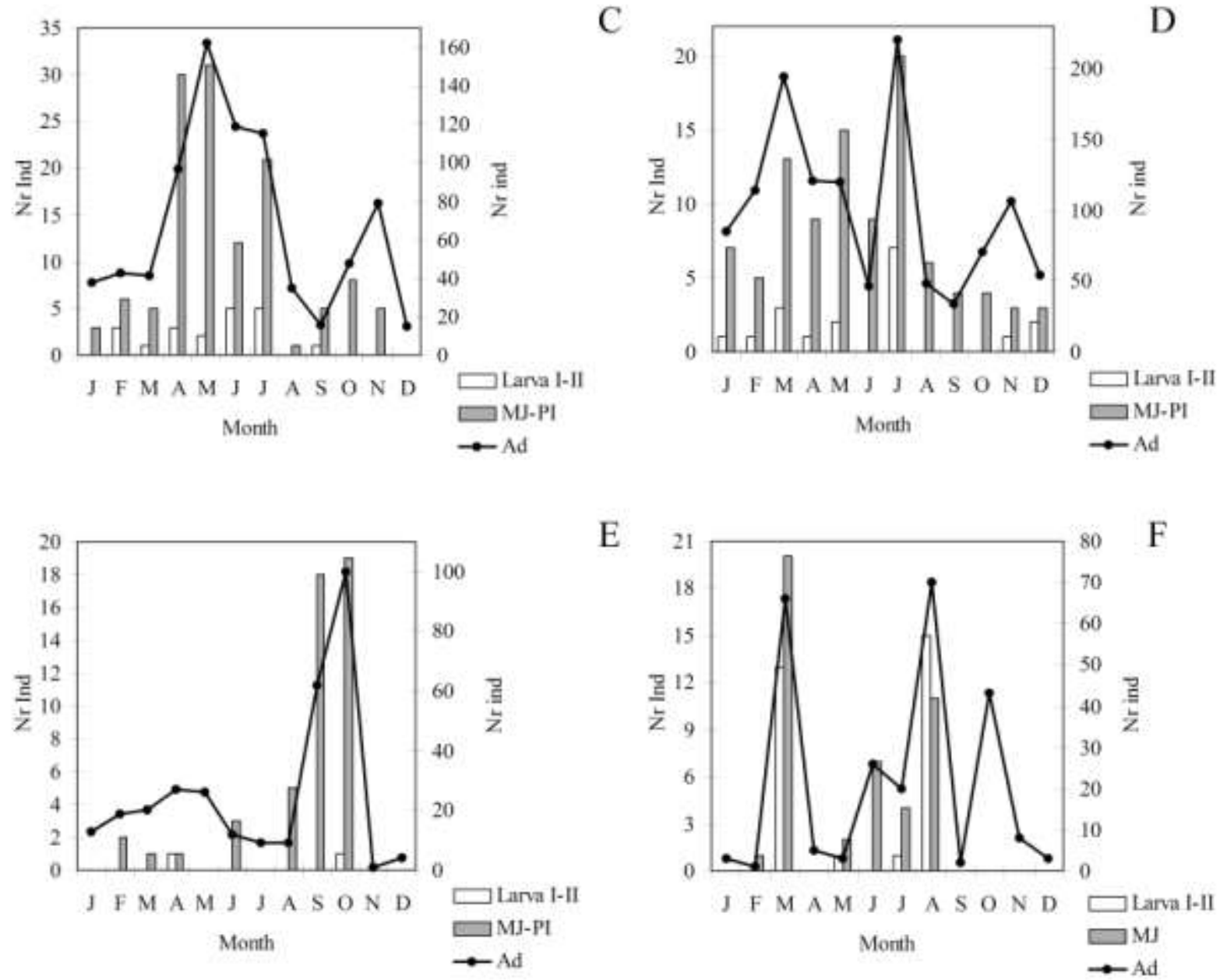

E

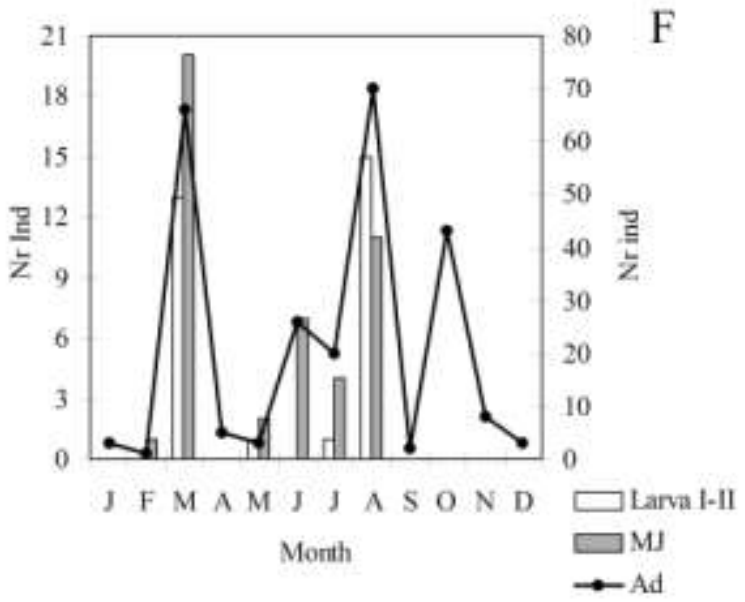

Fig. 3. Analysis of phenology of the five dominant species of Protura in Italy and Corsica. Monthly distribution of collections analyzed (A). Acerentomon gallicum (B). A. maius (C). A. italicum (D). A. microrhinus (E). Eosentomon transitorium (F). 